\title{
Diabetes tipo 2 y obesidad leve: tratamiento quirúrgico*
}

\author{
Dr. ENRIQUE LANZARINI S. ${ }^{1}$ \\ 1 Departamento de Cirugía Hospital Clínico Universidad de Chile. \\ Santiago, Chile.
}

\section{TRABAJO DE INGRESO}

\begin{abstract}
Surgical treatment in type 2 diabetes and mild obesity

Introduction: In the last years, type 2 diabetes mellitus (T2DM) and obesity have become a serious public health problem, behaving as epidemic diseases. There is great interest in exploring different options for treatment of T2DM in non-morbidly obese patients. Objective: To report parameters of glicemic control in patients with T2DM and mild obesity who underwent laparoscopic Roux-en-Y Gastric Bypass (RYGBP). Material and Methods: Prospective clinical trial that includes patients with T2DM with a Body Mass Index (BMI) between 30 and $35 \mathrm{~kg} / \mathrm{mt}^{2}$ who underwent laparoscopic RYGBP from July 2008 through October 2010. Results: Thirty-one patients were included in the study, 15 men and 16 women, with an average age of $48.7 \pm 8.6$ years. The average time since onset of T2DM was 5.8 years. The average postoperative follow-up is 30.4 months. The average preoperative blood glucose and glycosylated hemoglobin was $152 \pm 70 \mathrm{mg} / \mathrm{dl}$ and $7.7 \pm 2.1 \%$, respectively. All of them were using oral hypoglycemic agents, and 4 patients were insulin dependent. Only one patient had a postoperative complication (hemoperitoneum). At 36 months follow up the average BMI decreased to $24.7 \mathrm{Kg} / \mathrm{mt}^{2}$, all patients (31) showed improvement in their glycemic control, and 29 of them (93.6\%) met criteria for remission of T2DM in the last control. Conclusion: Laparoscopic RYGBP is a safe and effective procedure that improves glycemic control in patients with T2DM and mild obesity at mid-term follow up.
\end{abstract}

Key words: Tipe 2 diabetes, surgery, mild obesity.

\section{Resumen}

Introducción: En la actualidad la diabetes mellitus tipo 2 (DM2) y la obesidad representan un serio problema de salud, comportándose como enfermedades epidémicas. Existe un gran interés en explorar distintas opciones de tratamiento de la DM2 en pacientes que no tienen obesidad mórbida. Objetivo: Reportar los parámetros de control glicémico en pacientes con DM2 y obesidad leve sometidos a bypass gástrico (BPG) a 3 años de seguimiento. Metodología: Estudio clínico prospectivo que incluye pacientes con DM2 con un IMC entre 30 y $35 \mathrm{~kg} / \mathrm{m}^{2}$ que se sometieron a bypass gástrico laparoscópico desde julio de 2008 hasta octubre

*Recibido el 12 de agosto de 2012 y aceptado para publicación el 24 de septiembre de 2012.

El autor no refiere conflictos de interés.

Correspondencia: Dr. Enrique Lanzarini S. Santos Dumont 999, Santiago, Chile. elanzarini@hotmail.com 
de 2010 como tratamiento de su DM2. Resultados: Estudio clínico compuesto por 31 pacientes, 15 hombres y 16 mujeres, con una media de edad de 48 años. El tiempo de evolución promedio de la DM2 fue de 5,8 años. El promedio de seguimiento postoperatorio es 30 meses. El promedio de glicemia en el preoperatorio fue $152 \mathrm{mg} / \mathrm{dl}$, todos utilizaban hipoglicemiantes orales, y 4 pacientes utilizaban insulina. El promedio de hemoglobina glicosilada preoperatorio fue de 7,7\%. Sólo un paciente presentó una complicación postoperatoria (hemoperitoneo). A los 3 años de seguimiento el IMC promedio disminuyó a 24,7 kg/m², el 93,6\% cumplió criterios de remisión de la DM2 y el 6,4\% mostró mejoría. Conclusión: El bypass gástrico representa un procedimiento seguro y eficaz en el control glicémico de los pacientes con diabetes tipo 2 y obesidad leve a 3 años de seguimiento.

Palabras clave: Diabetes tipo 2, cirugía, obesidad leve.

\section{Introducción}

La incidencia y prevalencia de la Diabetes Mellitus tipo 2 (DM2) está en aumento constante, constituyendo una epidemia global, estimándose que para el año 2025 cerca de 300 millones de personas, estarán afectadas en el mundo ${ }^{1,2}$. En Chile, la prevalencia actual de Diabetes es 9,4\% según la Encuesta Nacional de Salud ${ }^{3}$, lo que representa un importante problema de salud pública, no sólo por su comportamiento epidemiológico, sino que también por la gravedad de sus complicaciones crónicas ${ }^{4}$, siendo en estos pacientes los eventos cardiovasculares la primera causa de muerte ${ }^{5}$, con un riesgo similar al de los pacientes coronarios ${ }^{6}$. El tratamiento actual de la DM2 se basa en cambios de estilo de vida, dieta, ejercicios y farmacoterapia con hipoglicemiantes orales o insulina, que logran un buen control en un número insuficiente de pacientes ${ }^{7}$. Además, siendo una enfermedad progresiva para la que no existe terapia que logre detener su curso natural ${ }^{8}$, en el transcurso del tiempo deben ir combinándose fármacos para lograr un buen control metabólico, lo que paradójicamente disminuye la adherencia y provoca un control deficiente de la enfermedad ${ }^{9}$; si bien es cierto han aparecido fármacos que se asocian a lenta progresión de la enfermedad, como las tiazolidinedionas, no están exentos de efectos adversos como aumento del riesgo de fracturas ${ }^{10,11} \mathrm{e}$ insuficiencia cardíaca $^{12}$. Otros fármacos, como análogos de GLP1 e inhibidores de DPP-4, que tendrían un rol protector sobre la célula beta, no cuentan aún con estudios a largo plazo de seguridad y eficacia; en este sentido, la FDA reporta la posibilidad de que los análogos de GLP-1 se pueden asociar a pancreatitis aguda y carcinoma medular de tiroides ${ }^{13}$.

Por otro lado, la experiencia acumulada en cirugía bariátrica, la ha consolidado como el tratamiento de elección para pacientes con obesidad mórbida, habiendo mostrado además estabilidad en la pérdida del exceso de peso a largo plazo y un control efectivo de las comorbilidades asociadas, con especial énfasis en la DM2, Hipertensión Arterial, Dislipidemia y Resistencia a la Insulina ${ }^{14}$. En el caso específico de la
DM2, un meta-análisis publicado el año 2004 mostró que la cirugía bariátrica produjo la remisión de esta enfermedad en un $76,8 \%$ de los pacientes ( $84 \%$ para bypass gástrico), con normalización de la glicemia, reducción de los niveles de insulina y de la hemoglobina glicosilada ${ }^{15}$. Este efecto se produce durante los primeros días o semanas post-cirugía, antes de una pérdida de peso significativa, lo que sugiere un efecto específico de los cambios anatómicos y funcionales post-qurúrgicos, y no sólo por la pérdida de peso y restricción de la ingesta de carbohidratos ${ }^{16,17}$. Por todo lo anterior, distintos grupos quirúrgicos hemos planteado la posibilidad de ampliar la indicación de cirugía bariátrica al grupo de pacientes con DM2 con obesidad leve (Índice de Masa Corporal (IMC) entre 30 y $35 \mathrm{~kg} / \mathrm{m}^{2}$ ) como tratamiento de la DM2, lo que se ha conocido como Cirugía Metabólica.

El objetivo de este trabajo es reportar los resultados en cuanto a control glicémico y baja de peso de pacientes con DM2 y obesidad leve sometidos a bypass gástrico con intención de tratar la DM2.

\section{Metodología}

Estudio clínico prospectivo no aleatorio que incluye pacientes con DM2 que consultaron espontáneamente en el Policlínico de Cirugía Bariátrica del Hospital Clínico de la Universidad de Chile, que fueron seleccionados de acuerdo con los siguientes criterios de inclusión:

1. DM2 diagnosticada por endocrinólogo de acuerdo a los criterios de la Asociación Americana de Diabetes (ADA).

2. IMC entre 30 y $35 \mathrm{~kg} / \mathrm{m}^{2}$.

3. Edad entre 18 y 65 años.

4. Hemoglobina glicosilada entre 7 y $9 \%$.

5. Péptido $\mathrm{C}>1 \mathrm{ng} / \mathrm{ml}$.

6. Estudio inmunológico negativo para diabetes autoinmune: anticuerpos anti-islote (ICA), antitirosinfosfatasa (IA2), anti-descarboxilasa del ácido glutámico (GADA) y anti-insulina (AAI).

7. Sin evidencia clínica de macroangiopatía.

8. Evaluación nutricional, psicológica y endocrinológica. 
Los pacientes que cumplieron los criterios de inclusión fueron presentados en un comité creado específicamente para evaluar a estos pacientes (Comité de Cirugía Metabólica), compuesto por cirujanos bariátricos, un endocrinólogo, nutriólogos, psiquiatra y psicólogos, que evaluaron en conjunto caso a caso, decidiendo la indicación quirúrgica. Todos los pacientes seleccionados firmaron un consentimiento informado para la cirugía y fueron sometidos a un bypass gástrico en el Departamento de Cirugía del Hospital Clínico de la Universidad de Chile, a partir de julio de 2008 hasta Octubre de 2010.

\section{Técnica quirúrgica}

Con el paciente en posición francesa (cirujano entre las piernas), se insertan 5 trocares bajo visión directa. Después de la separación del lóbulo hepático izquierdo, se confecciona pouch gástrico de 20 a $40 \mathrm{ml}$ aproximadamente, con suturas mecánicas. A continuación, se identifica el ángulo de Treitz, y se asciende asa yeyunal de $200 \mathrm{~cm}$ (asa biliopancreática), ante-cólica y se realiza anastomosis gastro-yeyunal mecánica calibrada con un sonda de 38F (12,7 mm) y Maxon ${ }^{\circledR}$ 3-0 sutura continua. Se asciende asa yeyunal de $100 \mathrm{~cm}$ (asa alimentaria) y se realiza entero-entero anastomosis con sutura mecánica y Maxon ${ }^{\circledR}$ 3-0 sutura continua, contigua a anastomosis previa. Utilizando azul de metileno, se realiza prueba de hermeticidad de ambas anastomosis. Sección yeyunal entre anastomosis quedando conformada la Y de Roux. Se dejó un tubo de drenaje a la anastomosis gastro-yeyunal.

\section{Seguimiento}

Se realizó un control clínico 1 semana después del alta, y control clínico y de laboratorio (glicemia en ayunas, hemoglobina glicosilada, péptido $\mathrm{C}$, insulinemia basal, perfil lipídico y hemograma) a los $3,6,9,12,18,24$ meses y luego cada 1 año después de la cirugía. Al mismo tiempo, los pacientes fueron controlados por el equipo de nutrición y endocrinología. Se definió como "Remisión" de la DM2 un nivel de glicemia en ayunas de $100 \mathrm{mg} / \mathrm{dl}$ o menos, hemoglobina glicosilada $<6 \%$ y la suspensión completa de medicamentos para la diabetes. "Mejoría" se definió como una disminución o la suspensión parcial de los medicamentos para la diabetes y/o la disminución de los niveles de glucosa en sangre en ayunas en $126 \mathrm{mg} / \mathrm{dl}$. Se consideró "Igual o Peor" para los pacientes que no cambiaron o aumentaron su dosis de medicamento para la diabetes, y/o no han mejorado sus niveles de glicemia en ayunas. Los datos recogidos fueron tabulados y analizados utilizando el software de Microsoft ${ }^{\circledR}$ Excel. Para el análisis estadístico se utilizó la prueba T de Student para grupos pareados, y para calcular el \% de fun- ción de célula beta y $\%$ de sensibilidad a la insulina se utilizó la fórmula HOMA 2 de célula $\beta$ del Centro para la Diabetes, Endocrinología y Metabolismo de la Universidad de Oxford.

\section{Resultados}

Serie prospectiva compuesta por 31 pacientes con DM2, 15 hombres y 16 mujeres, con una edad media de 48,7 $\pm 8,6$ años. El peso promedio de antes de la cirugía fue de $85,4 \pm 13,2 \mathrm{~kg}$, con un IMC promedio de $33,1 \pm 1,8 \mathrm{~kg} / \mathrm{m}^{2}$. (Tabla 1) El tiempo promedio de evolución de la DM2 fue de 5,8 años. El 39\% de los pacientes tenían hipertensión y $64,5 \%$ de ellos eran dislipidémicos. En cuanto al tratamiento de la DM2, el 53,6\% utilizaba un agente hipoglicemiante oral (HGO), 32,1\% utilizaba dos HGO, y el 14,3\% eran usuarios de la insulina, sola o en combinación con HGO. En cuanto al control metabólico preoperatorio, el promedio de glicemia en ayunas fue 152 $\pm 7 \mathrm{mg} / \mathrm{dl}$, la hemoglobina glicosilada (HbAlc) fue en promedio $7,7 \pm 2,1 \%$, el Péptido C $3,49 \pm 2,6 \mathrm{ng} /$ dl, el porcentaje de Sensibilidad a la Insulina $34 \%$ y el porcentaje de Secreción de Insulina por la célula beta $69 \%$. Todos los pacientes fueron sometidos a un bypass gástrico laparoscópico. Un paciente evolucionó con un hemoperitoneo en el postoperatorio que requirió re-exploración por vía laparoscópica, con una recuperación favorable. El promedio de estadía hospitalaria fue de 5 días (rango: 4-20 días). No hubo mortalidad en la serie. El seguimiento postoperatorio promedio fue 30 meses (rango: 12-40 meses). La pérdida de peso fue rápida en los primeros 6 meses, llegando a un IMC de $24,3 \pm 2,2 \mathrm{~kg} / \mathrm{m}^{2}$, y luego se mantuvo estable dentro de rango de normalidad hasta los 3 años con valor promedio de $24,7 \pm 1,2 \mathrm{~kg} / \mathrm{m}^{2}$ (p $<0,001$ ) (Figura 1). En cuanto a los parámetros de control metabólico, la glicemia en ayunas mostró una disminución significativa en los primeros 6 meses, a un promedio de $88,5 \pm 9,8 \mathrm{mg} / \mathrm{dl}$, y se mantuvo

Tabla 1. Características clínicas y demográficas

\begin{tabular}{|llcc|}
\hline & & $\mathbf{n}$ & $\mathbf{\%}$ \\
Sexo & Femenino & 16 & $51,6 \%$ \\
& Masculino & 15 & $48,4 \%$ \\
Edad (años) & Promedio & $48,7 \pm 8,6$ & \\
Peso (kg) & Promedio & $85,4 \pm 13,2$ & \\
IMC (kg/m $\left.{ }^{2}\right)$ & Promedio & $33,1 \pm 1,8$ & \\
Evolución DM2 & Promedio & 5,8 años & \\
Media seguimiento & & 30 meses & \\
Rango (meses) & & $12-40$ & \\
\hline
\end{tabular}




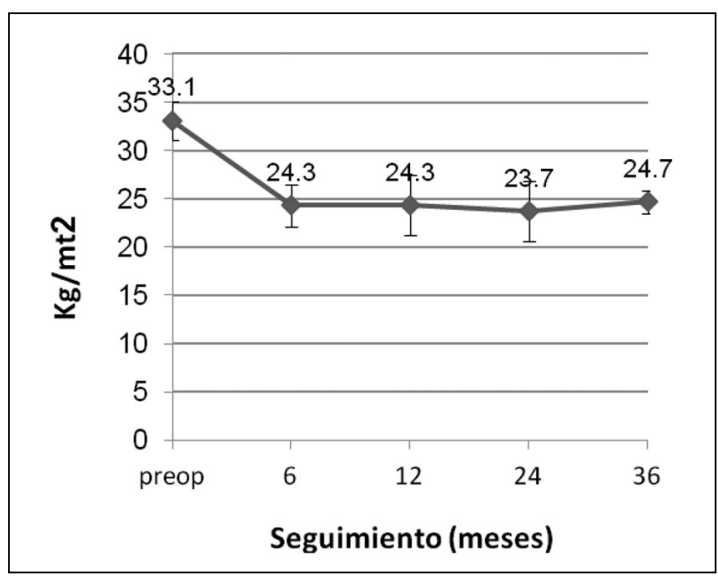

Figura 1. Índice de masa corporal.

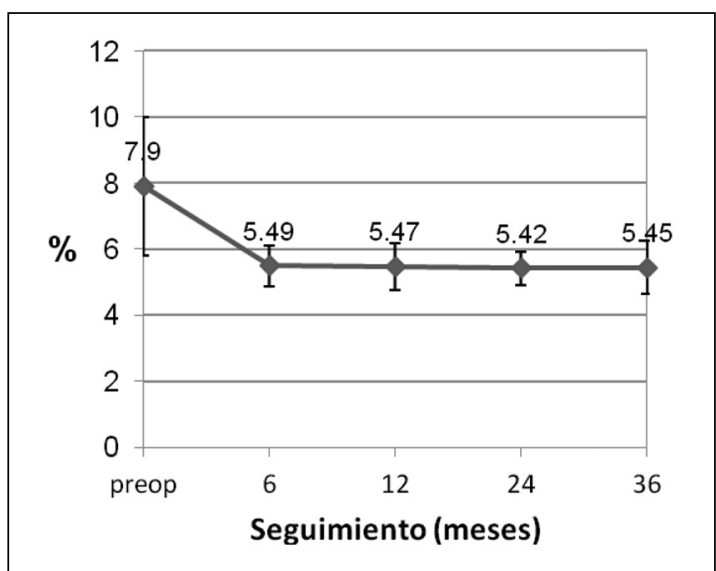

Figura 3. $\mathrm{HbA1c}$.

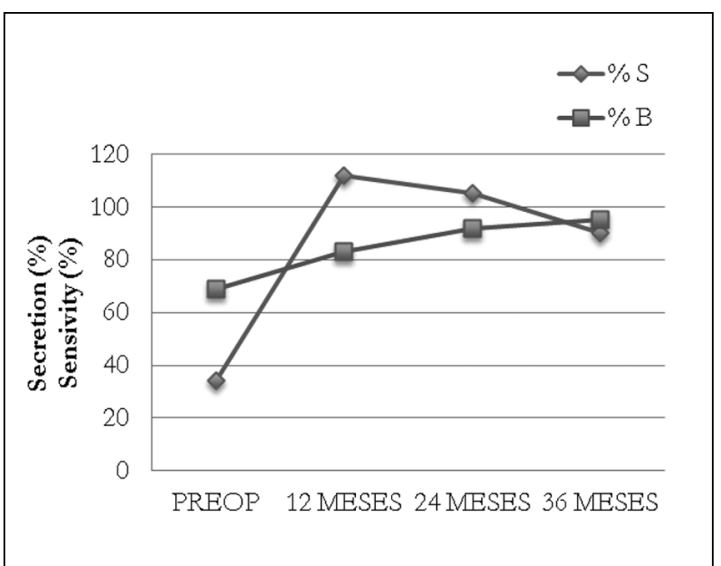

Figura 5. Porcentaje secreción célula B y porcentaje sensibilidad a la insulina.

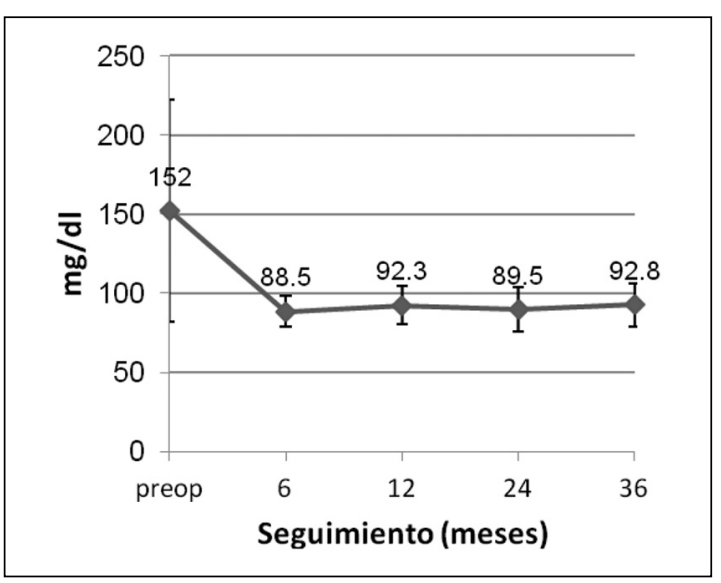

Figura 2. Glicemia.

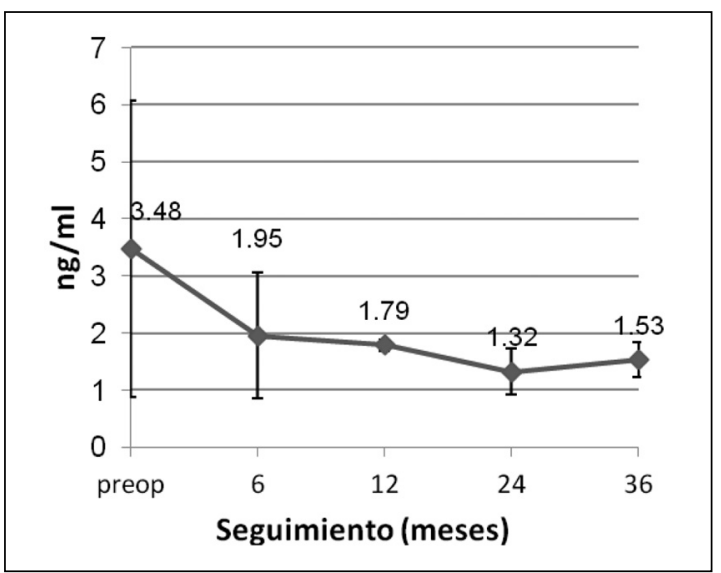

Figura 4. Péptido C.

dentro de rango de normalidad hasta los 3 años con promedio de $93 \pm 13,7 \mathrm{mg} / \mathrm{dl}(<0,001)$ (Figura 2). La hemoglobina glicosilada bajó a un promedio de $5,4 \pm 0,6 \%$ a los 6 meses, y también se mantuvo bajo la meta de control hasta los 3 años $(5,4 \pm 0,8 \%)$ (p $<0,003$ ) (Figura 3). El Péptido $\mathrm{C}$ disminuyó progresivamente hasta un promedio de $1,53 \pm 0,3 \mathrm{ng} /$ dl a los 3 años (Figura 4). La función secretora de la célula beta aumentó progresivamente hasta un 95\% a los 3 años de seguimiento, y la sensibilidad a la insulina aumentó hasta un $90 \%$ a la misma fecha. (p $<0,05$ ) (Figura 5). Utilizando los datos del último control, el 93,6\% de los pacientes diabéticos fueron considerados en remisión, y el $6,4 \%$ tuvieron una mejoría en su control metabólico. Ningún paciente fue considerado "igual o peor" en términos de control metabólico y el uso de la medicación. 


\section{Discusión}

Un control estricto en los niveles de glicemia en los pacientes diabéticos ha demostrado un beneficio importante en reducción de mortalidad y de complicaciones relacionadas ${ }^{7}$, sin embargo, el tratamiento actual logra estos objetivos en una pequeña proporción de pacientes ${ }^{17}$.

La cirugía bariátrica a través del tiempo ha demostrado una reducción significativa del exceso de peso, un control efectivo de las comorbilidades asociadas a obesidad, y una disminución significativa de la mortalidad a largo plazo ${ }^{18-22}$.

En cuanto a la diabetes, Buchwald en su metaanálisis del año 2004, compuesto por estudios de procedimientos bariátricos en pacientes con IMC $>35 \mathrm{~kg} / \mathrm{m}^{2}$, mostró una remisión general de $77 \%$, siendo de un $84 \%$ para $\mathrm{BPG}^{15}$. El año 2009 , el mismo autor en una revisión sistemática y nuevo metaanálisis de 621 estudios, que incluye 135.000 pacientes, encuentra que 103 estudios reportan remisión de la DM2 clínica y/o de laboratorio en un 78,1\% de los pacientes ${ }^{23}$. El estudio clásico S.O.S (Swedish Obese Subjects) demostró claramente la prevención y remisión sostenida de la DM2 en un grupo de 2.037 pacientes con obesidad severa que fueron sometidos a cirugía bariátrica en comparación con el grupo no quirúrgico a los 2 y 10 años de seguimiento ${ }^{24}$.

Hasta hace poco existía sólo un estudio prospectivo randomizado controlado que evaluó la cirugía bariátrica como tratamiento para la DM2, que comparó la banda gástrica ajustable con manejo médico, siendo la remisión de la DM2 de 73\% para el grupo quirúrgico versus $13 \%$ para el grupo no quirúrgico, a dos años de seguimiento ${ }^{25}$. Recientemente se han publicado dos trabajos prospectivos randomizados que comparan tratamiento médico versus quirúrgico en DM2. El primero, Mingrone, publica remisión en $75 \%$ para bypass gástrico, $95 \%$ para derivación bilio-pancreática y no hubo remisión en el grupo de manejo médico a 2 años de seguimiento en 60 pacientes diabéticos con índice de masa corporal $>35 \mathrm{~kg} / \mathrm{m}^{2}$ y hemoglobina glicosilada $>7 \%{ }^{26}$. Por su parte, Schauer, reporta en 150 pacientes con índice de masa corporal entre 27 y $43 \mathrm{~kg} / \mathrm{m}^{2}$ y diabetes no controlada, una reducción de hemoglobina glicosilada bajo $6 \%$ en un $42 \%$ para bypass gástrico, $37 \%$ para gastrectomía en manga y sólo $12 \%$ para el grupo con manejo médico intensivo. El uso de fármacos para disminuir la glicemia, lípidos y presión arterial se redujeron significativamente en los pacientes quirúrgicos a 12 meses de seguimiento ${ }^{27}$.

La alta tasa de remisión de la DM2 en pacientes con obesidad severa o mórbida sometidos a cirugía bariátrica, sugiere que la cirugía es el mejor tratamiento disponible para la diabetes en esta cohorte de pacientes, hecho que se ve reflejado en la Guía Clínica de la Asociación Americana de Diabetes "Estándares de Atención Médica en la Diabetes", publicadas el año 2009, que menciona el tratamiento quirúrgico en pacientes con diabetes mal controlada que tienen un IMC $>35 \mathrm{~kg} / \mathrm{m}^{2}$, y en IMC $<$ a $35 \mathrm{~kg} /$ $\mathrm{m}^{2}$ sólo bajo protocolos de investigación ${ }^{28}$.

Los mecanismos responsables de la remisión de la DM2 después de la cirugía son parcialmente conocidos y actualmente en estudio. Como hemos aprendido de los pacientes con IMC $>35 \mathrm{~kg} / \mathrm{m}^{2}$, hay mecanismos peso-dependientes cuyos efectos aparecen más tarde después de la cirugía y son proporcionales a la pérdida de peso, y existen mecanismos independientes del peso, cuyos efectos aparecen precozmente después de la cirugía y son dependientes del tipo de procedimiento quirúrgico (restrictivo puro, mal absortivo o mixto).

En relación a los mecanismos dependientes del peso, sabemos que la obesidad es el factor de riesgo más importante para el desarrollo y la progresión de la diabetes, a través del desarrollo de resistencia a la insulina, secreción de citoquinas inflamatorias, y una reducción sistemática de la masa de células beta pancreáticas. La pérdida de peso inducida por métodos tradicionales tiene un poderoso efecto en la prevención del desarrollo de la diabetes, mejora significativamente el control metabólico y reduce la mortalidad asociada ${ }^{29-31}$. En comparación con los métodos no quirúrgicos, la pérdida de peso inducida por la cirugía bariátrica es significativamente mayor $\mathrm{y}$ tiende a permanecer en el seguimiento a largo pla$\mathrm{zo}^{15}$. Los efectos beneficiosos de la cirugía bariátrica en el control de la diabetes, resistencia a la insulina, hipertensión y otras comorbilidades asociadas a la obesidad, parecen ser proporcionales a la pérdida de peso, e inversamente proporcional al tiempo de evolución de la diabetes ${ }^{32}$.

Los mecanismos independientes del peso son menos conocidos, pero hasta el momento es bastante claro que son múltiples, y la activación de éstos dependen de los cambios anatómicos y funcionales inducidos por cada procedimiento en particular ${ }^{18}$.

La teoría del Intestino Distal se basa en los cambios del tránsito intestinal inducidos por el BPG, Derivación Bilio-pancreática (DBP) y otros procedimientos, como la Interposición Ileal, en que los alimentos llegan precozmente a los segmentos distales del intestino, lo que genera una estimulación de las células epiteliales L del íleon, con producción de GLP-1 y PYY (péptidos anorexígenos con efecto incretina). Esto ocurre tempranamente después de la cirugía, antes de que se produzcan cambios significativos del peso corporal del paciente ${ }^{29}$. La teoría del Intestino Proximal se basa en la observación de que la exclusión del duodeno y yeyuno proximal obser- 
vada en el BPG, DBP, y procedimientos endoscópicos con instalación de un dispositivo endoluminal, mejora la tolerancia a la glucosa. Esta hipótesis fue probada experimentalmente por Rubino en ratones diabéticos tipo 2 sometidos a bypass duodeno yeyunal, lo que generó una mejora en la tolerancia a la glucosa, la cual fue reversible después de reiniciar el tránsito normal en el segmento intestinal excluido ${ }^{14}$. Otros mecanismos como la secreción de Ghrelina, los cambios en la microbiota intestinal y la mayor disponibilidad de ácidos biliares en el íleon, son menos conocidos y están actualmente bajo investigación.

La presencia de un efecto específico e independiente de la cirugía sobre la diabetes ha generado un nuevo campo de investigación sobre la patogenia de la DM2, y ha dirigido a grupos quirúrgicos a explorar la cirugía bariátrica desde el punto de vista metabólico, más que por sus resultados en pérdida de peso.

La experiencia publicada en el tratamiento quirúrgico de la diabetes en pacientes con IMC $<35 \mathrm{~kg} / \mathrm{m}^{2}$ es limitada. El número de pacientes tratados en cada experiencia es pequeña, la técnica quirúrgica utilizada para cada grupo es diferente y el seguimiento es escaso. Sin embargo, los resultados iniciales presentados por estos grupos quirúrgicos son en general favorables ${ }^{32,33,35}$.

Una revisión sistemática publicada el año 2010, que incluyó 16 estudios con 343 pacientes diabéticos con IMC $<35 \mathrm{~kg} / \mathrm{m}^{2}$ operados con distintas técnicas bariátricas, mostró remisión en el $85 \%$ del total de la muestra, con una media de seguimiento de 23 meses, siendo un $72,2 \%$ para las técnicas restrictivas, $97,7 \%$ para las mixtas (bypass gástrico) y $72,9 \%$ para las mal absortivas ${ }^{36}$.

En conclusión, se presentan los resultados iniciales de un protocolo de investigación en cirugía metabólica, en pacientes diabéticos con obesidad leve con regular control metabólico y reserva pancreática estimada a través de la medición del péptido $\mathrm{C}$. Se realizó BPG ya que después de años de experiencia en cirugía bariátrica, es un procedimiento seguro y eficaz, con una tasa de mortalidad baja $(0,16 \%$ a $0,40 \%$ ), similar a otros procedimientos quirúrgicos comunes como la colecistectomía laparoscópica ${ }^{21}$, con morbilidad en disminución constante, con una tasa reportada de $7,4 \%$, con reducciones importantes en las fugas anastomóticas, infecciones post operatorias y complicaciones respiratorias ${ }^{22}$. Estas reducciones son en su mayoría atribuidos a la estandarización de la técnica y el uso amplio de la cirugía laparoscópica. En segundo lugar, la reconstrucción con Y de Roux permite una exclusión del duodeno y yeyuno proximal y estimulación del intestino distal, con los beneficios mencionados previamente.
Los resultados iniciales son prometedores, con una tasa de remisión de la DM2 en un 93\% teniendo en cuenta el último control. No tenemos reportes de pérdida de peso exagerada o desnutrición en nuestra serie. De hecho, nuestros pacientes mostraron una estabilización del peso corporal en un IMC de $24 \mathrm{~kg} /$ $\mathrm{m}^{2}$ en promedio. Todos nuestros pacientes recibieron seguimiento con el equipo multidisciplinario, suplementación vitamínica oral y parenteral de vitamina B12, calcio y control endocrinológico. Al comparar nuestros resultados con los de otros grupos o revisiones sistemáticas, obtuvimos niveles de remisión más altos, creemos que esto se debe a que nuestros pacientes tienen menos tiempo de evolución de la DM2, utilizan menos fármacos hipoglicemiantes y tienen mejor reserva pancreática de células beta.

Teniendo en cuenta los datos publicados y nuestra experiencia inicial, el bypass gástrico podría ser una opción terapéutica válida en un grupo seleccionado de pacientes diabéticos con obesidad leve. Sin embargo, la experiencia es limitada y actualmente no tenemos evidencia para recomendar su uso generalizado en diabéticos con IMC $<35 \mathrm{~kg} / \mathrm{m}^{2}$ frente al tratamiento médico disponible actualmente. El desarrollo de protocolos clínicos controlados con los mismos criterios y la misma técnica quirúrgica en centros de alto volumen con experiencia en cirugía bariátrica ofrecerá mejor calidad de evidencia, lo que permitirá definir qué grupo de pacientes diabéticos y en qué punto de evolución de la enfermedad recibirán un beneficio a largo plazo del tratamiento quirúrgico.

\section{Agradecimientos}

A los Drs. Attila Csendes, Italo Braghetto, Luis Gutiérrez, Rodolfo Lahsen, Verónica Araya, Karin Papapietro, Pablo Olguín, Hanns Lembach, Juan C. Molina y Pedro Cuevas.

A las nutricionistas Andrea Riffo y Emma Díaz. A las psicólogas Denisse Montt y Luciana Marín.

\section{Referencias}

1. Zimmet P. The burden of type 2 diabetes: are we doing enough? Diabetes Metab. 2003;29:9-18.

2. Wild S, Roglic G, Green A, Sicree R, King H. Global Prevalence of Diabetes. Estimates for the year 2000 and projections for 2030. Diabetes Care 2004;27:1047-53.

3. Encuesta Nacional de Salud 2009-2010. Ministerio de Salud. Gobierno de Chile.

4. Donnelly R, Emslie Smith A, Gardner I, Morris A. Complications of Diabetes. BMJ 2000;320:1062-6.

5. Geiss L, Herman W, Smith P. Mortality in non insulin- 
dependent diabetes."Diabetes in America” $2^{\text {nd }}$ edition. National diabetes data group. National Health Institutes. National Institute of Diabetes and Digestive and Kidney Diseases NIH Publication. 1995;95:1468.

6. Haffner S, Lehto S, Rönnemaa T, Pyörälä K, Laakso M. Mortality from Coronary Heart Disease in subjects with Type 2 Diabetes and in nondiabetic subjects with or without prior myocardial infarction. N Eng J Med. 1998;339:229-34.

7. UK Prospective Diabetes Study Group. Effect of intensive blood glucose control with metformin on complications in overweight patients with type 2 diabetes (UKPD 34). Lancet 1998;352:854-65.

8. Heine R, Diamant M, Mbanya J-C, Nathan D. Management of Hyperglycemia in Type 2 Diabetes: The End of Recurrent Failure? BMJ 2006;333:1200-4.

9. Dailey G, Kim M, Lian J. Patient Compliance and Persistence with Anti-Hyperglycemic Therapy: Evaluation of a Population of Type 2 Diabetic Patients. J Int Med Res. 2002;30:71-9.

10. Kahn S, Haffner S, Heise M, Herman W, Holman R, Jones N, et al, for the ADOPT Study Group. Glycemic Durability of Rosiglitazone, Metformin, or Glyburide Monotherapy. N Engl J Med. 2006;355:2427-43.

11. Dormandy J, Charbonnel B, Eckland D, Erdmann E, Massi-Benedetti M, Moules I, et al., On behalf of the PROactive Investigators. Secondary Prevention on Macrovascular Events in Patients with Type 2 Diabetes in the PROactive Study (PROspective pioglitAzone Clinical Trial In macroVascular Events): A Randomised Controlled Trial. Lancet 2005;366:1279-89.

12. Lago R, Singh P, Nesto R. Congestive Heart Failure and Cardiovascular Death in Patients with Prediabetes and Type 2 Diabetes Given Thiazolidinediones: A Meta-Analysis of Randomised Clinical Trials. Lancet 2007;370:1129-36.

13. Parks M, Rosenbraugh $\mathrm{C}$. Weighing risks and benefits of liraglutide-the FDA's review of a new anidiabetic therapy. N Eng J Med. 2010;362:774-7.

14. Rubino F, Gagner M. Potential of Surgery for Curing Type 2 Diabetes Mellitus. Ann Surg. 236:554-9.

15. Buchwald H, Avidor Y, Braunwald E, Jensen MD, Pories W, Fahrbach K, et al. Bariatric surgery: a systematic review and meta-analysis. JAMA 2004;292:1724-37.

16. Schauer PR, Ikramuddin S, Gourash W, Ramanathan $\mathrm{R}$, Luketich J. Outcomes after laparoscopic roux-enY gastric bypass for morbid obesity. Ann Surg. 2000; 232:515-29.

17. Pories WJ, Swanson MS, MacDonald KG, Long SB, Morris PG, Brown BM, et al. Who would have thought it? An operation proves to be the most effective therapy for adult-onset diabetes mellitus. Ann Surg.1995 222:339-50.

18. Rubino F, Schauer P, Kaplan L, Cummings D. Metabolic surgery to treat type 2 diabetes: clinical outcomes and mechanisms of action. Annu Rev Med.
2010;61:393-411.

19. Sjöstrom L, Narbro K, Sjöström CD, Karason K, Larsson B, Wedel H, et al. Effects of bariatric surgery on mortality in Swedish obese subjects. N Eng J Med. 2007;357:741-52.

20. Sjöstrom L, Gummesson A, Sjöström CD, Narbro K, Peltonen, Wedel H, et al. Effects of bariatric surgery on cancer incidence in obese patients in Sweden (Swedish Obese Subjects Study): a prospective controlled intervention trial. Lancet Oncol. 2009;10:653-62.

21. Adams T, Gress R, Smith S, Halverson R, Simper S, Rosamond W, et al. Long-term mortality after gastric bypass surgery. N Engl J Med. 2007;357:753-61.

22. Christou N, Sampalis J, Liberman M, Look D, Auger S, McLean L. Surgery decreases long-term mortality, morbidity and health care use in morbidly obese patients. Ann Surg. 2004;240:416-24.

23. Buchwald H, Estok R, Fahrbach K, Banel D, Jensen $\mathrm{MD}$, Pories WJ, et al. Weight and type 2 diabetes after bariatric surgery: systematic review and meta-analysis. Am J Med. 2009;122:248-56.

24. Sjöstrom L, Lindroos AK, Peltonen M, Torgerson J, Bouchard C, Carlsson B, et al. Lifestyle, diabetes, and cardiovascular risk factors 10 years after bariatric surgery. N Engl J Med. 2004;351:2683-93.

25. Dixon JB, O'Brien PE, Playfair J, Chapman L, Schachter LM, Skinner S et al. Adjustable gastric banding and conventional therapy for type 2 diabetes: a randomized controlled trial. J Am Med Assoc. 2008;299:316-23.

26. Mingrone G, Panunzi S, De Gaetano A, Guidone C, Laconelli A, Leccesi L. Bariatric Surgery versus Conventional Medical Therapy for Type 2 Diabetes. N Engl J Med. 2012;366:1577-85.

27. Schauer P, Kashyap S, Wolski K, Brethauer S, Kirwan J, Pothier C. Bariatric Surgery versus Intensive Medical Therapy in Obese Patients with Diabetes. N Engl J Med. 2012;366:1567-76.

28. American Diabetes Association. Standards of medical care in diabetes 2009. Diabetes Care. 2009; 32:S13-61.

29. Dixon J. Obesity and Diabetes: The Impact of Bariatric Surgery on Type-2 Diabetes. World J Surg. 2009; 33:2014-21.

30. Williamson D, Thompson T, Thun M. Intentional weight loss and mortality among overweight individuals with diabetes. Diabetes Care 2000;23:1499-504.

31. Pi-Sunyer X, Blackburn G, Brancati F. Reduction in weight and cardiovascular disease risk factors in individuals with type 2 diabetes: one-year results of the look AHEAD trial. Diabetes Care 2007;30:1374-83.

32. Pontiroli A, Pizzocri P, Librenti M, Vedani P, Marchi M, Cucchi E, et al. Laparoscopic adjustable gastric banding for the treatment of morbid (grade 3) obesity and its metabolic complications: a three-year study. J Clin Endocrinol Metab. 2002;87:3555-61.

33. Lee W, Wang W, Lee Y, Huang M, Ser K, Chen J. Effect of laparoscopic mini-gastric bypass for type 2 diabetes 
mellitus: comparison of BMI $>35$ and $<35 \mathrm{~kg} / \mathrm{m}^{2}$. J Gastrointest Surg. 2008;12:945-52.

34. DePaula A, Macedo A, Rassi N, Machado C, Schraibman V, Silva L, Halpern A. Laparoscopic treatment of type 2 diabetes mellitus for patients with a body mass index less than 35. Surg Endosc. 2008;22:706-16.

35. Ramos AC, Galvão Neto MP, de Souza YM, Galvão M, Murakami AH, Silva AC, et al. Laparoscopic Duodenal-
Jejunal Exclusion in the Treatment of Type 2 Diabetes Mellitus in Patients with BMI $<30 \mathrm{~kg} / \mathrm{m}^{2}$ (LBMI). Obes Surg. 2009;19:307-12.

36. Fried M, Ribaric J, Buchwald J, Svacina S, Dolezalova K, Scopinaro N. Metabolic Surgery for the Treatment of Type 2 Diabetes in Patients with BMI $<35 \mathrm{~kg} / \mathrm{mt}^{2}$ : An Integrative Review of Early Studies. Obes Surg. 2010;20:776-90.

\section{INFORME}

En primer lugar, quisiera agradecer a la Sociedad de Cirujanos de Chile la oportunidad de informar el trabajo de ingreso del Dr. Enrique Lanzarini a nuestra Sociedad. El Dr. Lanzarini nos presenta un interesante estudio de cohorte de pacientes diabéticos con obesidad leve, sometidos a bypass gástrico.

Como el Dr. Lanzarini señala, la diabetes mellitus tipo 2 es uno de los principales factores de riesgo de enfermedades cardiovasculares, las que constituyen la primera causa de muerte en Chile y el mundo. $\mathrm{Su}$ incidencia ha aumentado significativamente en los últimos años, de acuerdo a la última Encuesta Nacional de Salud 2009-2010 afecta al 9,4\% de la población en nuestro país. Del mismo modo, la prevalencia de la obesidad, factor de riesgo de diabetes tipo 2, ha aumentado en nuestro país y el mundo entero.

La cirugía de la obesidad ha demostrado buenos resultados en términos de baja de peso a largo plazo en pacientes con obesidad mórbida. Esto tiene como consecuencia una mejoría significativa en la calidad de vida de estos pacientes, su expectativa de vida y el control de enfermedades asociadas. En pacientes diabéticos con obesidad mórbida estudios retrospectivos demuestran remisión de la enfermedad en 60 a $90 \%$ de los pacientes dependiendo de las técnicas empleadas y de la baja de peso asociada. Esto ha motivado considerar la cirugía como una alternativa de control en pacientes diabéticos con obesidad leve e incluso con sobrepeso. Resultados preliminares de estudios prospectivos controlados han demostrado buenos resultados en el control a corto plazo de procedimientos como bypass gástrico, gastrectomía vertical y banda gástrica ajustable metabólico de diabetes tipo 2 con IMC 27 a 35 con mal control metabólico comparado con tratamiento médico estándar. Los pacientes sometidos a cirugía presentan una mayor reducción de los niveles de hemoglobina glicosilada a uno y dos años con un menor uso de medicamentos.

Los resultados que el Dr. Lanzarini presenta son consistentes con estos estudios observando una dis- minución significativa de los niveles de hemoglobina glicosilada de $7,9 \%$ a 5,47\% en un año, remisión de la enfermedad de $93 \%$ de los pacientes y una disminución del índice de masa corporal de 33,1 a $24,2 \mathrm{~kg} / \mathrm{m}^{2}$. Este análisis incluye pacientes con buen y mal control metabólico de la diabetes donde el rango de hemoglobina glicosilada preoperatoria es de 5,1 a $12,6 \%$. Del mismo modo el Dr. Lanzarini presenta buenos resultados en el control de otras enfermedades asociadas de alta prevalencia en estos pacientes como es la dislipidemia con reducción significativa de los niveles plasmáticos de colesterol y triglicéridos hasta 3 años de seguimiento.

Esta información constituye un muy buen punto de partida en la evaluación del rol de la cirugía en este tipo de pacientes donde sin duda existen muchas preguntas aún por responder. Es interesante evaluar resultados a largo plazo desde el punto de vista del control de la diabetes, desarrollo de complicaciones y por sobre todo expectativa de vida. Del mismo modo, es importante evaluar el balance costo beneficio del tratamiento quirúrgico a largo plazo considerando el alto costo en salud que representan los pacientes obesos con diabetes tipo 2 .

El Dr. Lanzarini es un joven y destacado cirujano nacido en la histórica ciudad de Chillán. Cursó sus estudios primarios y de enseñanza media en la ciudad de Los Angeles desde donde se trasladó a estudiar Medicina a la Universidad de Concepción. El Dr. Lanzarini se formó como cirujano general en la sede Norte de la Universidad de Chile donde actualmente trabaja y ha desarrollado una interesante y prolífica línea de investigación de cirugía de la obesidad y metabólica.

Los resultados del estudio que el Dr. Enrique Lanzarini nos presenta es sin duda un aporte al desarrollo y crecimiento de la cirugía en nuestro país y ciertamente su incorporación como miembro titular un orgullo para la Sociedad de Cirujanos de Chile. Su ingreso representa también un ejemplo a seguir por las nuevas generaciones de cirujanos de nuestro país. Por estos motivos recomiendo sin 
reparos la incorporación del Dr. Lanzarini a nuestra Sociedad; agradezco nuevamente la oportunidad de participar en esta sesión. Al Dr. Lanzarini le invito e insto a seguir trabajando en el desarrollo y crecimiento de la docencia e investigación en nuestra noble especialidad con el absoluto apoyo y respaldo de la Sociedad de Cirujanos de Chile, muchas gracias.

Dr. Alex Escalona

Departamento de Cirugía Hospital Clínico Pontificia Universidad Católica

Santiago, Chile 